\title{
Endothelial cells of human colorectal cancer and healthy colon reveal phenotypic differences in culture
}

\author{
Vera Simone Schellerer ${ }^{1,2}$, Roland Siegfried Croner $^{1}$, Kristina Weinländer $^{2}$, Werner Hohenberger ${ }^{1}$, Michael Stürzl ${ }^{2}$ \\ and Elisabeth Naschberger ${ }^{2}$
}

Antiangiogenic drugs have been used successfully for the treatment of colorectal cancer (CRC). Viable tumor endothelial cells (TEC) and normal endothelial cells (NEC) of uninvolved colon tissue of the same patient have not been available to optimize treatment strategies in vitro. Therefore, our target was to establish a protocol for the isolation of TEC and NEC. These cells were isolated with very high purity via magnetic cell sorting of tissue samples obtained from CRC and healthy colon of eight patients. TEC and NEC expressed CD31, CD105, VE-cadherin, VCAM-1, ICAM-1 and E-selectin, formed capillaries in basal membrane extract and were able to take up acetylated low-density lipoprotein. They were negative for podoplanin, CD45, CD68 and cytokeratin-20 indicating blood vessel endothelial lineage. Intense staining of von Willebrand factor (VWF) was observed in five of eight NEC cultures, whereas vWF was absent or only slightly expressed in all TEC cultures in vitro. Low intracellular concentration of VWF was also detected in TEC and NEC at the tissue level. This demonstrated that differences exhibited by TEC and NEC in vivo are stably perpetuated in culture. The isolated cultures may provide a useful in vitro model to elucidate epigenetic effects on angiogenesis in cancer and to optimize antiangiogenic therapy.

Laboratory Investigation (2007) 87, 1159-1170; doi:10.1038/labinvest.3700671; published online 17 September 2007

KEYWORDS: angiogenesis; colorectal cancer; endothelial cells; von Willebrand factor

The formation of new blood vessels from pre-existing vasculature (angiogenesis) is key to progression and metastasis of solid tumors. ${ }^{1-4}$ Without adequate vascular supply, solid tumors do not grow beyond a size of $1-2 \mathrm{~mm}$. For further progression, angiogenesis is needed to compensate the lack of nutrients and oxygen. ${ }^{5}$

Angiogenesis is initiated by proangiogenic factors, like vascular endothelial growth factor and basic fibroblast growth factor, which are released by the tumor cells and activate resting endothelial cells (EC) to migrate, proliferate, differentiate and finally to form new blood vessels. ${ }^{5-7}$ The rapid proliferation of tumor endothelial cells (TEC) and the persistent proangiogenic stimulation leads to the formation of incomplete and irregular vessels with fenestrations, irregular blood flow and increased permeability compared to normal vasculature. ${ }^{2,8,9}$ TEC are clearly different from normal endothelial cells (NEC) as indicated by the expression of specific 'tumor endothelial markers' and cytogenetical abnormalities. ${ }^{10,11}$
Colorectal cancer (CRC) is one of the leading cancers in the western countries with 500000 deaths worldwide per year. ${ }^{12}$ Almost a third of all patients already have metastatic disease at diagnosis. ${ }^{13}$ Nearly $50 \%$ of all diagnosed CRC patients will subsequently develop metastasis with mostly fatal prognosis. ${ }^{13}$ More than a century, surgery has played the major role in curative treatment of CRC. The treatment of choice for advanced disease is surgery combined with adjuvant chemotherapy. ${ }^{12}$ Recently, Hurwitz et $a l^{14}$ presented promising results using bevacizumab, a potent antiangiogenic drug, in combination with chemotherapeutics for treatment of advanced disease. Therefore, clinicians start to focus on TEC as a reasonable target for antiangiogenic strategies.

So far, meaningful in vitro model systems are missing to optimize treatment strategies of antiangiogenic therapy in CRC.

The aim of this study was to establish an easy protocol for the isolation of pure, viable EC from human CRC and

\footnotetext{
'Department of Surgery, Friedrich-Alexander University, Erlangen, Germany and ${ }^{2}$ Division of Molecular and Experimental Surgery, Department of Surgery, Friedrich-Alexander University, Erlangen, Germany

Correspondence: Professor M Stürzl, PhD, Division of Molecular and Experimental Surgery, Department of Surgery, Friedrich-Alexander University of Erlangen-Nuremberg, Schwabachanlage 10, Erlangen 91054, Germany. E-mail: michael.stuerzl@chir.imed.uni-erlangen.de 
healthy colon tissue. These cells may provide a promising platform for the comparative analysis of both cell types at the molecular and cell biological level.

\section{MATERIALS AND METHODS Tissue Collection}

Tissues of tumor and healthy colon were obtained from patients undergoing standard surgical procedure for primarily diagnosed CRC at the Department of Surgery. Tumors were histopathologically characterized according to the Union International Contre le Cancer (UICC). The samples collected for EC isolation included two UICC-Stages I, one UICC-Stage II, two UICC-Stages III and three UICC-Stages IV CRC. The mean age of patients was 66 years. Clinical parameters of the patients are listed in Table 1 . The procedure was approved by the Local Ethics Committee and all patients provided written informed consent.

\section{EC Isolation}

For isolation of EC the resected specimen was placed in ice-cold Hank's balanced salt solution (HBSS; PAA, Colbe, Germany) supplemented with $1 \times$ penicillin/streptomycin (PAA) and $250 \mu \mathrm{g} / \mathrm{ml}$ amphotericin B (PAA) for transportation. Pieces of $0.5-3 \mathrm{~g}$ (depending on tumor size) were cut from the center of the malignancy by the clinical pathologist and used for isolation of TEC. Morphologically visible hypoxic or necrotic parts of the tumor were avoided. Uncompromised healthy colon tissue was cut at a safety distance of minimum $10 \mathrm{~cm}$ apart the tumor and was used for isolation of NEC.

The tissues were washed four times with ice-cold HBSS supplemented with $1 \times$ penicillin/streptomycin and $250 \mu \mathrm{g} /$ $\mathrm{ml}$ amphotericin $\mathrm{B}$ and were minced into approximately $10 \mathrm{~mm}^{3}$ pieces with scissors. The pieces were digested with 17100 U/g Collagenase II (Biochrom AG, Berlin, Germany) in $5 \mathrm{ml}$ EC basal medium-2 supplemented with $0.5 \%$ fetal calf serum (EBM-2-0.5\% FBS; Cambrex, Verviers, Belgium) for $1 \mathrm{~h}$ in an atmosphere of $5 \% \mathrm{CO}_{2}$ and $37^{\circ} \mathrm{C}$ using a rotating mixer. Later, the tissues were squeezed through a metal mesh with a mesh size of less than $1 \mathrm{~mm}$ and cells were harvested by centrifugation $\left(500 \mathrm{~g}, 5 \mathrm{~min}, 20^{\circ} \mathrm{C}\right)$. Cells were cultivated in endothelial cell culture medium (EBM-2-MV) in T-25 culture flasks (Nunclon, Nunc, Wiesbaden, Germany), which were coated for at least $2 \mathrm{~h}$ with $1.5 \%$ bovine skin gelatine, type B (Sigma-Aldrich Chemie, Deisenhofen, Germany) in phosphate-buffered saline (PBS; Biochrom AG). Medium was changed every second day until colonies were visible.

\section{Magnetic Cell Sorting}

Five to seven days after initial seeding, EC were positively selected for CD31 by magnetic cell sorting (MACS) using MS separation columns (Miltenyi Biotech, Bergisch Gladbach, Germany). To this goal, cells were washed once with PBS and 1-2 ml Accutase (PAA) was added until cells were completely detached. Following the manufacturer's instructions a positive selection for EC using the CD31 MicroBead Kit (Miltenyi) was performed. Cells were passed sequentially over two separation columns to reach higher purity. EBM-2-MV was used for the final washing step and elution from the column. Cells were cultivated in $1.5 \%$ gelatine-coated $(40 \mathrm{~mm})$ dishes (Nunc) until confluence. In case of significant $(>10 \%)$ contamination by non-EC additional cycles of MACS were performed. The first confluent T-25 flask of pure EC was designated as passage 0 . One passage was defined by a split ratio of 1:4.

\section{Cell Culture}

Isolated EC were cultivated in $1.5 \%$ gelatine precoated culture flasks with EBM-2-MV. Medium change was performed every second day. Confluent cells were washed once with PBS, detached by using $1 \times 0.5 \mathrm{~g} / 1$ trypsin and $0.2 \mathrm{~g} / \mathrm{l}$ ethylenediaminetetraacetic acid in HBSS (Trypsin-EDTA; PAA, Colbe, Germany) for $2-3 \mathrm{~min}$ and passaged by a ratio of maximal 1:3.

\section{Immunocytochemistry}

For all stainings the cells were cultured on gelatine-coated LabTek chamber slides (Nunc) until they reached confluence and were subsequently fixed and stained. For VCAM-1, ICAM-1 and E-selectin staining the cells were starved for $14 \mathrm{~h}$ in EBM-2-0.5\% FBS and stimulated in the same medium with $300 \mathrm{U} / \mathrm{ml}$ tumor necrosis factor- $\alpha$ (TNF- $\alpha$; Roche, Mannheim, Germany) for $8 \mathrm{~h}$ (VCAM-1) or with $200 \mathrm{U} / \mathrm{ml}$ interleukin-1 $\beta$ (IL-1 $\beta$, Roche) for $14 \mathrm{~h}$ (ICAM-1, E-selectin) before fixation. For the VE-cadherin staining the cells were plated on $5 \mu \mathrm{g} / \mathrm{cm}^{2}$ fibronectin (BD Biosciences, Heidelberg, Germany) instead of gelatine. All slides were then washed once with PBS and fixed in ice-cold ethanol for at least $20 \mathrm{~min}$. The cells were rehydrated and incubated for $2 \mathrm{~h}$ with anti-CD31 (1:50, DakoCytomation, Hamburg, Germany, clone JC70A), anti-CD105 (1:200, DakoCytomation, clone SN6h), anti-von Willebrand factor (vWF) (1:200, DakoCytomation, clone F8/86), anti-CD45 (1:10, DakoCytomation, clone T29/33), anti-cytokeratin-20 (CK-20, 1:10, DakoCytomation, clone KS 20.8), anti-VCAM-1 (1:10, DakoCytomation, clone 1.4C3), anti-CD68 (1:100, DakoCytomation), anti-podoplanin (1:200, Bender MedSystems, Vienna, Austria), anti-ICAM-1 (1:20, R\&D Systems, Wiesbaden-Nordenstadt, Germany, clone BBIG-I1 11C8), anti-VE-cadherin (1:2, Santa Cruz, Heidelberg, Germany) or anti-E-selectin (1:40, R\&D Systems, clone BBIG-E4 5D11). Subsequently, rabbit-anti-mouse immunoglobulin (1:50, DakoCytomation) was added for $45 \mathrm{~min}$ followed by APAAP mouse (1:50, DakoCytomation) for another $45 \mathrm{~min}$. Staining was developed with Fuchsin Chromogen (DakoCytomation). Nuclei were counterstained with Gill-III hematoxylin (Merck KG, Darmstadt, Germany). Slides were mounted with Aqueous Mount (Zytomed Systems, Berlin, Germany). Stained sections and cells were photographed using a Sony 3CCD color video camera (Sony Corporation, Munich, Germany) 
Table 1 Clinical parameters of colorectal carcinoma patients $(n=8)$ included in the study

\begin{tabular}{lcccc}
\hline UICC & I & II & III & IV \\
\hline No. of patients & 2 & 1 & 2 & 3 \\
\hline Gender & $F^{a}$ & $M^{b}$ & $F$ & $M$ \\
& F & & $F$ & $M$ \\
& & & & F
\end{tabular}

\begin{tabular}{|c|c|c|c|c|}
\hline \multirow[t]{3}{*}{ Age } & 65 & 61 & 72 & 67 \\
\hline & 77 & & 66 & 70 \\
\hline & & & & 51 \\
\hline \multirow[t]{3}{*}{ Tumor invasion } & $\mathrm{T} 2$ & T3 & T3 & T3 \\
\hline & T3 & & $\mathrm{T} 2$ & T3 \\
\hline & & & & T4 \\
\hline \multirow[t]{3}{*}{ Lymph node status } & No & No & N2 & N1 \\
\hline & No & & N2 & $\mathrm{N} 2$ \\
\hline & & & & $\mathrm{N} 1$ \\
\hline \multirow[t]{3}{*}{ Distant metastasis } & $M x$ & $M x$ & $M x$ & M1 \\
\hline & $M x$ & & $M x$ & M1 \\
\hline & & & & M1 \\
\hline \multirow[t]{3}{*}{ Grading } & G2 & G2 & G2 & G3 \\
\hline & $\mathrm{G} 2$ & & G3 & $\mathrm{G} 2$ \\
\hline & & & & G3 \\
\hline \multirow[t]{3}{*}{ Localization } & Rectum & Cecum & $C^{c}$. ascendens & Rectum \\
\hline & Rectum & & C. ascendens & C. ascendens \\
\hline & & & & Cecum \\
\hline
\end{tabular}

${ }^{\mathrm{a}}$ Female.

${ }^{\mathrm{b}}$ Male.

${ }^{\mathrm{c}}$ Colon.

mounted on a Leitz Aristoplan microscope (Leica, Solms, Germany). Isotype controls were performed using an antimouse IgG1 antibody (R\&D Systems) in identical protein concentrations as the primary antibody. Each staining was performed for at least three different cultures of both, TEC and NEC. CD31 staining was routinely performed for all cultures.

\section{Binding of Ulex europaeus lectin and Uptake of Acetylated-Low-Density Lipoprotein}

Cells were cultured on gelatine-coated LabTek chamber slides (Nunc), incubated for $1 \mathrm{~h}$ with $5 \mu \mathrm{g} / \mathrm{ml}$ Ulex europaeus lectin, FITC-conjugated (Sigma-Aldrich) or $2.5 \mu \mathrm{g} / \mathrm{ml}$ acetylated-low-density lipoprotein (ac-LDL)/Alexa488-labeled (Invitrogen, Karlsruhe, Germany), washed three times with Tris-buffered saline and fixed in formalin for $10 \mathrm{~min}$. Nuclei were counterstained with DAPI $(1 \mu \mathrm{g} / \mathrm{ml}$; Invitrogen/ Molecular Probes) for $1 \mathrm{~min}$. Slides were mounted with fluorescent mounting medium and incorporation of ac-LDL or binding of lectin was visualized using a Leica Aristoplan Microscope (Leica) equipped with Leica IM50 software (Leica). Exposure times were kept constant for each channel.

\section{FACS Analysis}

Cells were harvested by Accutase treatment for the minimal time required for detachment and resuspended in PBS supplemented with $5 \%$ FCS and $0.1 \% \mathrm{NaN}_{3}$ (FACS-PBS). A total of $1 \times 10^{5}$ cells were incubated at $4{ }^{\circ} \mathrm{C}$ with a mouse monoclonal anti-CD31 antibody (1:100; Southern Biotechnology, AL, USA). After $45 \mathrm{~min}$, the cells were washed with PBS and stained with an Alexa 488-conjugated goat anti-mouse antibody (1:350; Invitrogen) for $30 \mathrm{~min}$ at $4^{\circ} \mathrm{C}$ in the dark. The cells were washed twice with PBS and resuspended in $300 \mu \mathrm{l}$ FACS-PBS. Flow cytofluorometry was performed using a FACSCalibur with CellQuest Pro software (BD Biosciences, CA, USA). Isotype control:mouse IgG1 antibody (1:500, R\&D Systems). For evaluation of the purity of the cells the background staining results observed in isotype controls were subtracted from the signals obtained in TEC and NEC stainings.

\section{Capillary Formation in Basement Membrane Extract}

Cells were starved in EBM-2-0.5\% FCS for $24 \mathrm{~h}$ at $80 \%$ confluency. Twelve-well plates were coated with $200 \mu$ l Cultrex basement membrane extract (reduced growth factor content, phenol red free) (R\&D Systems) per well for $30 \mathrm{~min}$ at $37^{\circ} \mathrm{C}$. In parallel, 12-well plates were coated with gelatine as described above. A total of 20000 cells per well were seeded on the plates and were either untreated or stimulated with $10 \mathrm{ng} /$ $\mathrm{ml}$ vascular endothelial growth factor (R\&D Systems) and $10 \mathrm{ng} / \mathrm{ml}$ basic fibroblast growth factor (Roche) in the starving medium for $24 \mathrm{~h}$. Capillary formation was photographed using a Zeiss Axiovert microscope (Zeiss, Jena, Germany) connected to an Olympus digital camera (Olympus, Munich, Germany).

\section{Survival Test}

Isolated EC ( $>95 \%$ ) were tested for their long-term viability by sequential passaging until arrest of growth. Total numbers of passages were determined for each culture.

\section{Western Blot Analysis}

Western blot from RIPA lysates was performed as described previously ${ }^{15,16}$ with the following exceptions: transfer was performed at $100 \mathrm{~mA}$ for $18 \mathrm{~h}$. The following primary antibodies were used for $1 \mathrm{~h}$ : polyclonal rabbit anti-human vWF antibody (1:3000, Abcam, Cambridge, UK) and 
mouse anti-human GAPDH (1:40 000, Chemicon/Millipore, Schwalbach, Germany). For the detection ECL mouse IgG and ECL rabbit IgG (both 1:5000, GE Healthcare, Munich, Germany) were used. Western blot was developed using ECL reagents (GE Healthcare).

\section{Immunohistochemistry}

CD31- and vWF-staining in formalin-fixed, paraffinembedded sections from tumor and normal tissues was performed as described previously. ${ }^{15,17,18}$ In brief, antigen retrieval was carried out using Target Retrieval Solution $\mathrm{pH}$ 6.1. Mouse monoclonal APAAP system was used as a detection system. The primary antibodies were incubated for $1 \mathrm{~h}$ and diluted as follows: CD31 (1:50), vWF (1:75). Staining was developed using Liquid Permanent Red. All reagents were purchased from DakoCytomation. Stained sections were quantitatively evaluated by three independent persons. The numbers of CD31- and vWF-positive vessels were counted in 10 optical fields (magnification $\times 870$ ) of each section. Mean values were calculated for tumor tissues and normal tissues. Subsequently, the ratio of the mean number of vWF-positive and CD31-positive vessels was calculated for tumor and normal tissues. The differences were evaluated by Student's $t$-test.

\section{Statistical Analysis}

Student's $t$-test was performed using SPSS 15.0 for windows software.

\section{RESULTS \\ Procedure for the Isolation of Pure EC from CRC and Normal Colon Tissue}

Tissues of CRC and healthy colon were collected from resected unfixed specimens of patients undergoing surgery for CRC (Figure 1a). Tumors were identified (Figure 1a, left, square) and the specimen for TEC isolation was resected from the central part of the tumor (Figure 1a, right, circle) to exclude contamination of TEC with NEC. Resection of apparently hypoxic or necrotic areas was avoided (Figure 1a, right, arrow). After several washing steps the tissues were minced in small pieces and digested by Collagenase II. For completing the dispartment, tissues were mechanically dissociated by mincing through a metal mesh. The single cell suspensions were cultivated in gelatine-precoated culture flasks. Within 5-7 days, cells with heterogeneous morphology arose, most likely representing a mixture of fibroblasts, muscle cells, epithelial cells and EC (Figure 1b).

For isolation of EC CD31- and CD105-MicroBeads were tested. Using CD105-MicroBeads frequently a contamination by non-EC was observed whereas purification with CD31MicroBeads specifically enriched EC and resulted in pure cultures (Figure 1c). CD31-FACS analysis revealed a purity of the isolated TEC and NEC of more than 93\% (corrected by background subtraction) as compared to commercially available HUVEC (94.68\%) and the negative control (CRC cell line DLD-1, 0\%) (Figure 1d). The whole isolation procedure is described in detail in Table 2. Using this procedure pairwise TEC and NEC could be isolated from CRC and healthy colon tissues of eight patients.

\section{TEC and NEC Show a Blood Vessel EC Phenotype}

The isolated cells displayed an EC-like cobble stone morphology and numerous mitoses (data not shown). Characterization of the isolated EC was performed by several different ways. All TEC and NEC formed capillaries on basement membrane extract (Figure 2, capillary formation), incorporated ac-LDL (Figure 2, ac-LDL uptake) and bound Ulex europaeus lectin (Figure 2, Ulex europaeus). Ac-LDL absorption and Ulex europaeus lectin binding were not observed in DLD-1 control cells (Figure 2, controls). In addition, isolated TEC and NEC expressed the EC markers CD31, CD105 and VE-cadherin (Figure 2, CD31, CD105 and VE-cadherin). Control stainings of the cells with isotype antibodies for CD31, CD105 or using staining system in the absence of a primary antibody were negative (Figure 2, controls).

Inducible adhesion molecules such as ICAM-1 (Figure 3a and c), VCAM-1 (Figure $3 \mathrm{~g}$ and i) and E-selectin (Figure $3 \mathrm{~m}$ and o) were expressed by TEC and NEC upon stimulation with inflammatory cytokines. HUVEC were used as a positive control (Figure $3 \mathrm{e}, \mathrm{k}$ and $\mathrm{q}$ ). Expression of the adhesion molecules was not detected in TEC, NEC or HUVEC without stimulation (Figure 3b, d, f, h, j, l, n, p and r).

\footnotetext{
Figure 1 Pure tumor endothelial cells (TEC) can be isolated from colorectal carcinoma by CD31-magnetic cell sorting (MACS). (a) Pieces of tumor up to $3 \mathrm{~g}$ were cut from a non-hypoxic, non-necrotic area of the central part (area of resection marked by circle; invasive margin marked by dashed line; necrotic area shown by arrow). Tissue for the isolation of normal endothelial cells (NEC) has been removed at a distance of about $10 \mathrm{~cm}$ from the tumor margin (dashed line). (b) Tissues were enzymatically digested by Collagenase II and mechanically dispersed. The resulting single cell suspension was seeded in gelatinecoated culture flasks. After 5-7 days phenotypically different cells arose. Scale bar corresponds to $100 \mu \mathrm{m}$. (c) Endothelial cells isolated from healthy colon and colorectal cancer (CRC) were positively selected using CD31-MicroBeads. The yield of endothelial cells was monitored by immunocytochemical staining of the CD31 surface antigen before and after MACS. Positive staining is visualized by pink color (arrows). Counterstaining was performed with hematoxylin (blue color). Scale bar corresponds to $100 \mu \mathrm{m}$. (d) CD31-FACS analysis of tumor (TEC) and normal endothelial cell (NEC) cultures ( $n=3$ ) in comparison to HUVEC as a positive and DLD-1 as a negative control. The relative percentages of CD31-positive cells (peak with line only) are given in the diagram and correspond to the cells in gate M1. The isotype antibody control peak is displayed by a peak shaded in gray. Results were corrected by subtraction of the results obtained in the stainings with the isotype control antibody.
} 
a

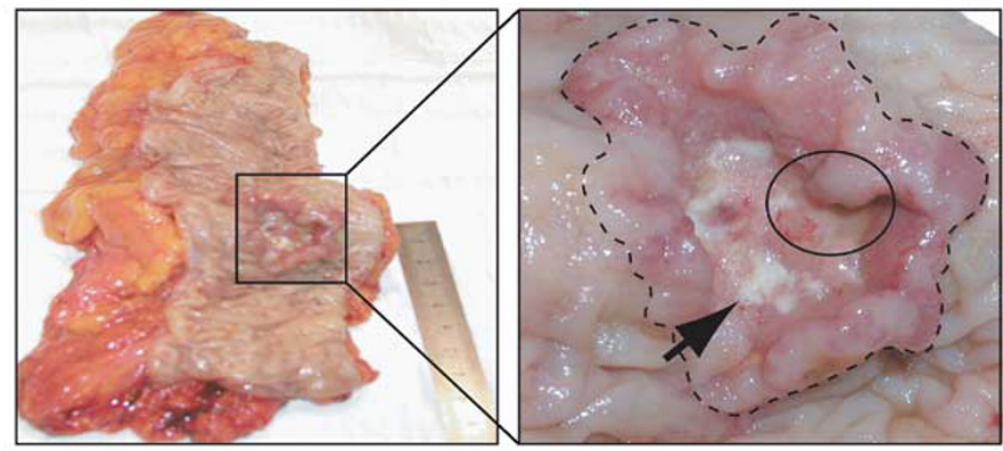

b
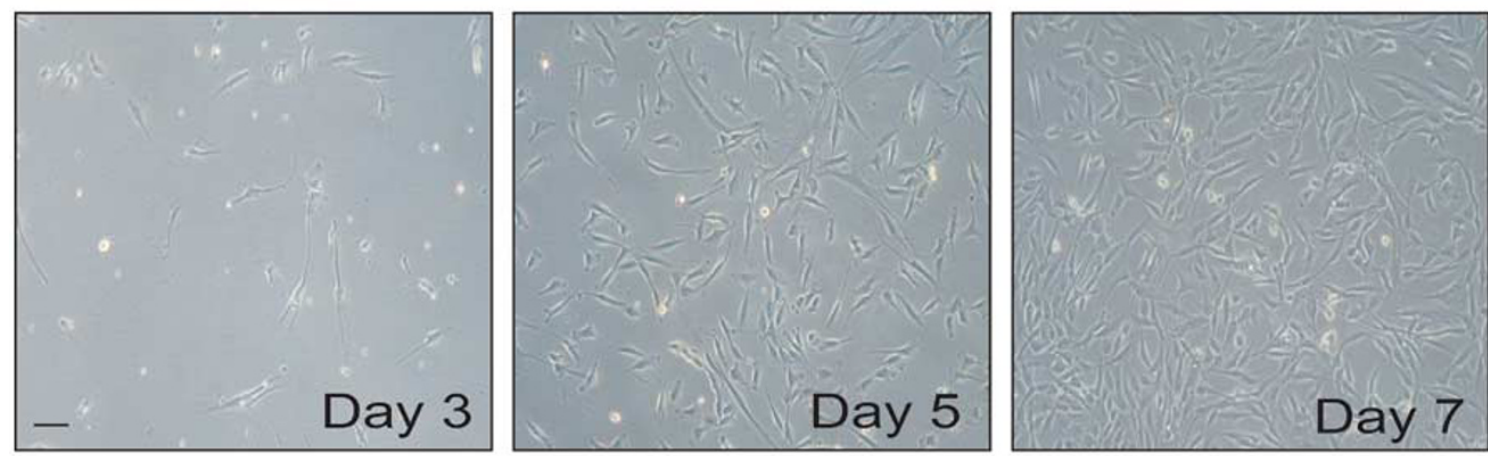

C
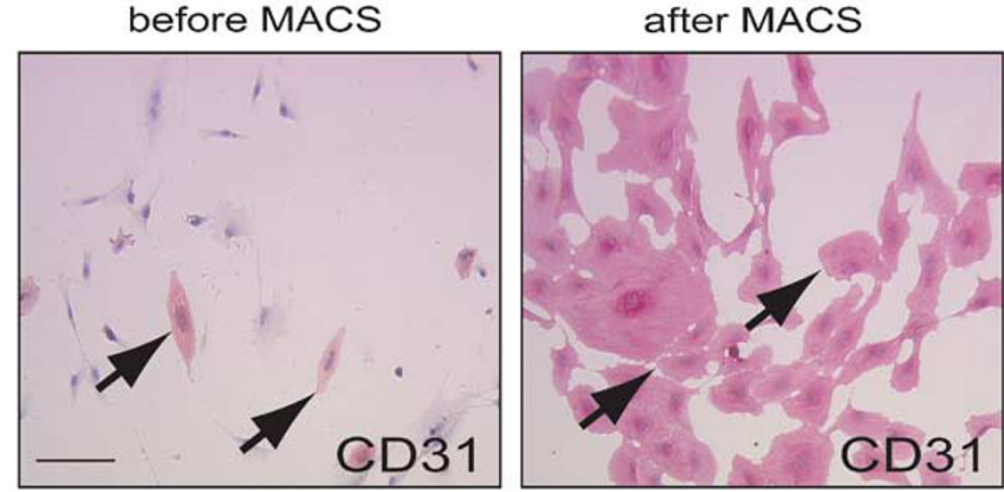

d
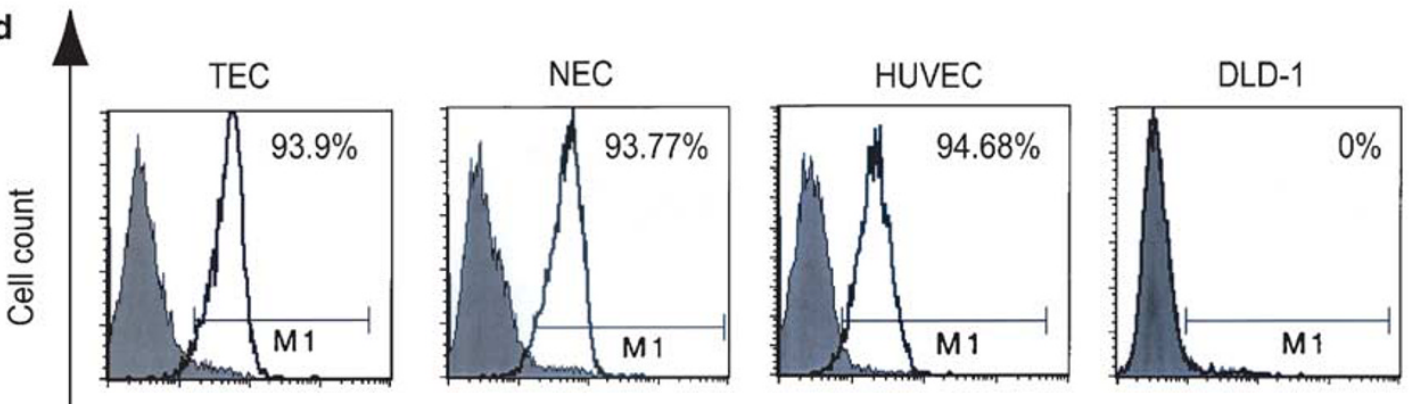

CD31 


\section{Table 2 Flow chart of the isolation procedure}

I. 1. Collect tissues in ice-cold HBSS supplemented with $1 \times$ Penicillin/ Streptomycin and $250 \mu \mathrm{g} / \mathrm{ml}$ amphothericin B (HBSS-P/S/A)

2. Wash tissue with HBSS-P/S/A

3. Mince tissue in $10 \mathrm{~mm}^{3}$ pieces

4. Digest with $17100 \mathrm{U}$ Collagenase $\mathrm{II} / \mathrm{g}$ tissue, $1 \mathrm{~h}, 5 \% \mathrm{CO}_{2}, 37^{\circ} \mathrm{C}$

5. Filter suspension through a metal mesh (mesh size $<1 \mathrm{~mm}$ )

6. Centrifuge at $500 \mathrm{~g}$ for $5 \mathrm{~min}$ at $20^{\circ} \mathrm{C}$

7. Cultivate pellet in $1.5 \%$ gelatine-coated T-25 flask with EBM-2-MV until confluent rates of $70 \%$

II. 1. Detach cells with accutase

2. Filter through $30 \mu \mathrm{m}$ pre-separation filter (Miltenyi)

3. Centrifuge at $300 \mathrm{~g}, 4^{\circ} \mathrm{C}, 10 \mathrm{~min}$

4. Discard supernatant

III. 1. Resuspend cells in $60 \mu$ l MACS buffer

2. Incubate with $20 \mu \mathrm{l} \mathrm{CD} 31$ MicroBeads and $20 \mu \mathrm{l} \mathrm{FcR} \mathrm{blocking}$ reagent for $15 \mathrm{~min}$ at $6-12^{\circ} \mathrm{C}$

3. Wash with $1 \mathrm{ml}$ MACS buffer

4. Centrifuge at $200 \mathrm{~g}, 4^{\circ} \mathrm{C}, 10 \mathrm{~min}$

5. Discard supernatant

6. Equilibrate MACS separation column with $500 \mu$ l MACS buffer

7. Resuspend pellet in $1 \mathrm{ml}$ MACS buffer and apply onto column

8. Wash column with $3 \times 500 \mu$ l MACS buffer

9. Equilibrate a second MACS column with $500 \mu$ l MACS buffer

10. Apply CD31-positive fraction of first column onto second column

11. Wash column with $2 \times 500 \mu \mathrm{l}$ MACS buffer and $1 \times 500 \mu \mathrm{l}$ medium

12. Flush positive fraction by using the plunger

13. Culture cells in $1.5 \%$ gelatine-coated $4 \mathrm{~mm}$ dish

14. In case of contamination by non-endothelial cells repeat MACS

I: dissection of tissues, II: preparation of single cell suspension, III: MACS-purification of CD31-positive endothelial cells.
Contamination by other cells was excluded by staining of CD45 (hematopoietic cells; Figure 4a and b), CD68 (monocytes/macrophages; Figure $4 \mathrm{~d}$ and e) and cytokeratin-20 (CK-20, colonic epithelial cells; Figure $4 \mathrm{~g}$ and $\mathrm{h}$ ), which were always negative in the TEC and NEC cultures. In contrast, the respective controls such as Jurkat cells, THP-1 and DLD-1 were clearly positive (Figure 4, controls, arrows). The lymphendothelial cell associated marker podoplanin was not detected in TEC and NEC suggesting that the isolated EC cultures were derived from blood vessel EC (Figure $4 \mathrm{j}$ and $\mathrm{k}$ ). In contrast, purified lymphatic endothelial cells (LEC), which were used as a control, were clearly positive for podoplanin (Figure 4l).

Isolated EC could be maintained up to 7.5 passages before growth arrest (data not shown).

\section{vWF is Differentially Expressed in TEC and NEC In Vitro and In Vivo}

Inflammation is an important process in CRC pathogenesis. vWF is an EC-associated factor, which is released by the cells after inflammatory activation. To investigate differences, which may be maintained in EC isolated from inflammatory CRC compared to non-inflammatory healthy colon tissues, the expression of vWF was analyzed in the TEC and NEC cultures, respectively. Interestingly, in the eight TEC cultures no or only a weak intracellular signal of vWF could be detected (Figure 5a, right panel, TEC). In contrast, robust expression of vWF was observed in five of the eight NEC, which were isolated from the same patients as the TEC (Figure 5a, left panel, NEC, arrows). Western blot analysis confirmed that intracellular vWF concentration is lower in TEC as compared to NEC (Figure 5b).

In the next step, it was investigated whether differential expression of vWF can be observed in TEC and NEC in vivo. Therefore, we stained CD31 and vWF in tissue samples of the five patients whose cultures showed differential vWF expression (Figure 5c). CD31- and vWF-positive vessels were counted for all the patients in 10 optical fields by three independent persons. The central part of the tumor was evaluated because the resected specimens for the isolation of EC were obtained from the center of the tumor but not from the invasive margin (compare Figure 1a, circle). In the normal

\footnotetext{
Figure 2 The isolated tumor endothelial cell (TEC) and normal endothelial cell (NEC) cultures show endothelial cell lineage. Pure endothelial cell populations (>93\%) of TEC and NEC were characterized by different assays. For capillary formation TEC and NEC were seeded onto an extract of extracellular basement membrane and were stimulated with angiogenic growth factors. HUVEC were used as a positive control. For uptake of acetylated low-density lipoprotein (ac-LDL), TEC and NEC were incubated with Alexa488-labeled acetylated LDL (green fluorescent signal, arrows) for $1 \mathrm{~h}$ and were counterstained by DAPI (blue fluorescent signal). TEC and NEC cultures were incubated with FITC-conjugated Ulex europaeus-lectin for $1 \mathrm{~h}$ and the signal (green, arrow) was visualized using epifluorescence microscopy. Counterstaining was performed with DAPI (blue fluorescence). Negative controls of ac-LDL and Ulex europaeus lectin binding have been performed with the colorectal carcinoma cell line DLD-1. Immunocytochemical staining of TEC and NEC was performed using CD31, CD105 and VE-cadherin antibodies. Positive staining is visualized by pink color (arrows). Counterstaining was performed with hematoxylin (blue color). For CD31 and CD105 isotype staining controls are displayed. As a negative control for VE-cadherin the staining was performed without primary antibody. Scale bars correspond to $100 \mu \mathrm{m}$ (capillary formation), $10 \mu \mathrm{m}$ (ac-LDL uptake), $100 \mu \mathrm{m}$ (CD31, CD105), $10 \mu \mathrm{m}$ (Ulex europaeus) and $50 \mu \mathrm{m}$ (VE-cadherin).
} 

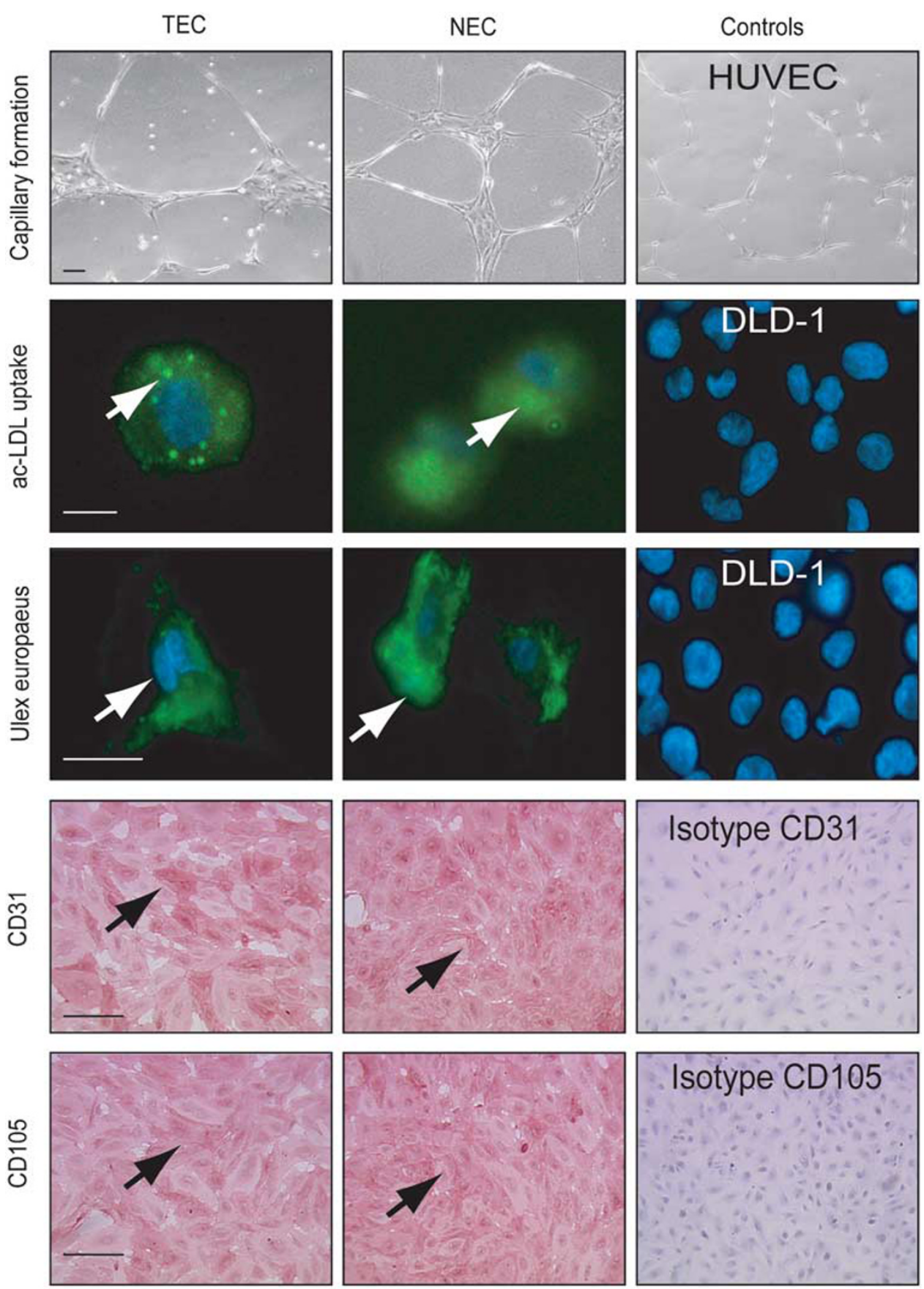

Isotype CD105
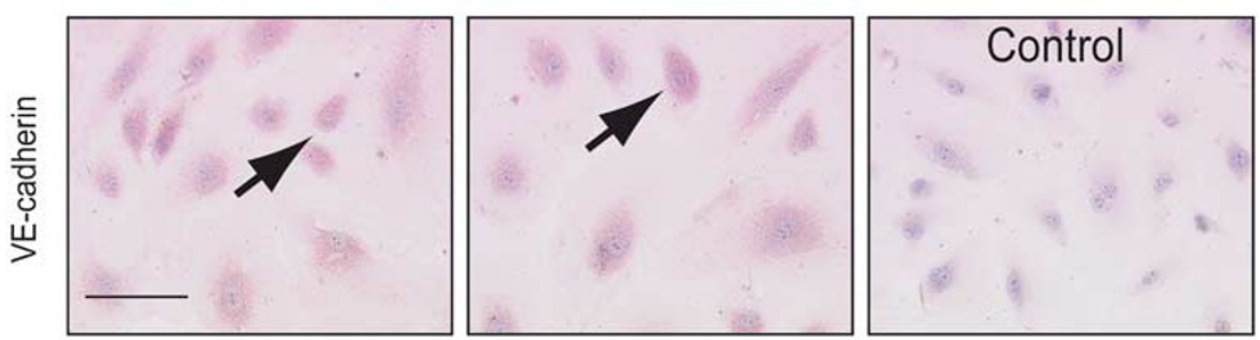


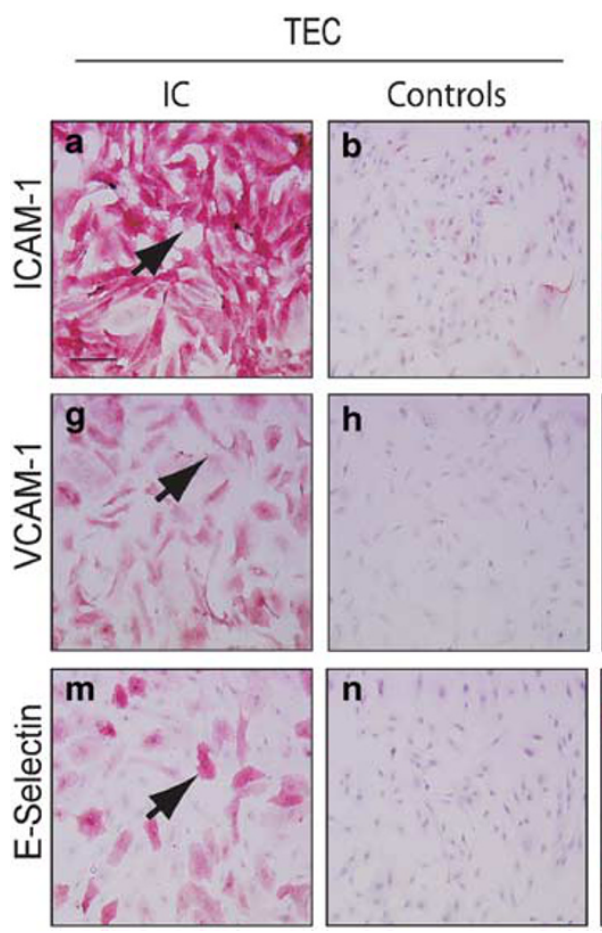

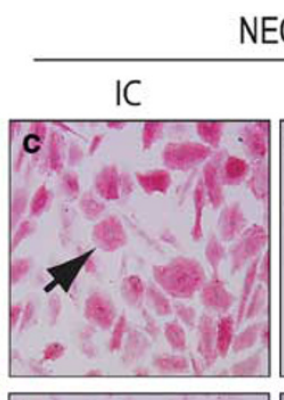

NEC
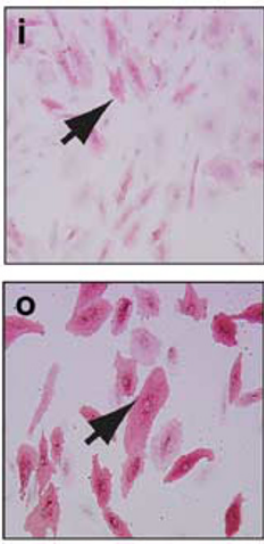
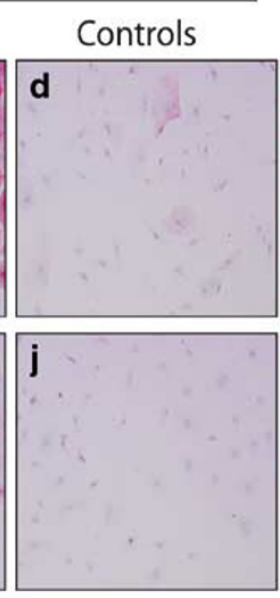

$\mathbf{p}$
HUVEC
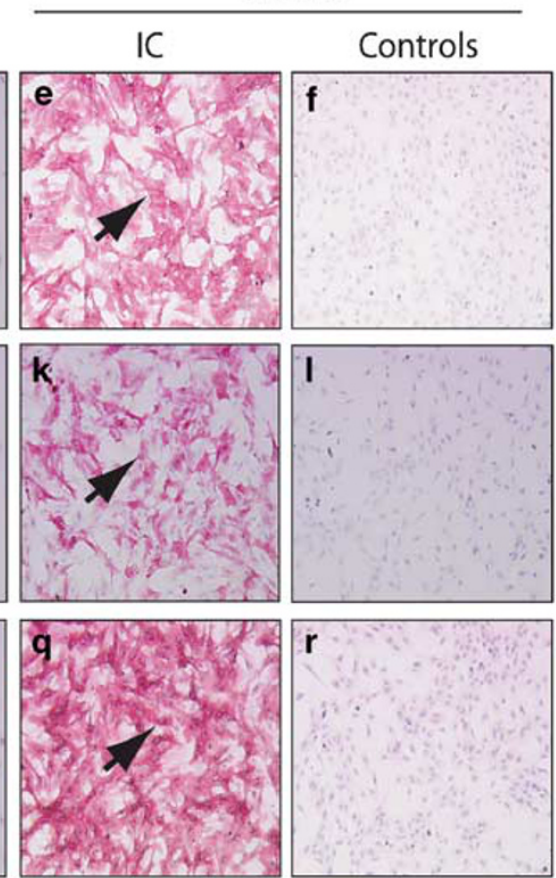

Figure 3 The isolated tumor endothelial cells (TEC) and normal endothelial cells (NEC) express the inducible endothelial cell adhesion molecules ICAM-1, VCAM-1 and E-selectin. TEC and NEC were immunocytochemically stained for (a and $\mathbf{c}$ ) ICAM-1, (g and $\mathbf{i})$ VCAM-1 and ( $\mathbf{m}$ and $\mathbf{o})$ E-selectin after stimulation with IL-1 $\beta$ (ICAM-1, E-selectin) or TNF- $\alpha$ (VCAM-1). HUVEC were used as a positive control (e, $\mathbf{k}$ and $\mathbf{q})$. Unstimulated (b, h and $\mathbf{n})$ TEC, (d, $\mathbf{j}$ and $\mathbf{p})$ NEC and (f, I and $\mathbf{r}$ ) HUVEC cultures were used as negative controls for each staining. Positive staining is visualized by pink color (arrows). Cells were counterstained with hematoxylin (blue color). Scale bar corresponds to $100 \mu \mathrm{m}$.

tissue sections the vessels in the submucosa were counted. Statistical analysis revealed that the average number of vWF positive morphologically detectable vessels per optical field was highly significant $(P=0.001)$ lower in the tumor (Figure $5 \mathrm{c}$; tumor vWF; $3.85 \pm 2.42$ vessels per optical field) as compared to the normal tissue (Figure 5c; mucosa vWF; $26.4 \pm 4.12$ vessels per optical field). This was confirmed by normalization of the results for the number of CD31-positive vessels (Figure 5c; tumor vWF/CD31-ratio: 0.113; mucosa vWF/CD31-ratio: 0.758).

Finally, we investigated whether an inflammatory tumor micromilieu which is commonly associated with CRC may induce the lower intracellular vWF levels in TEC. Therefore, NEC where exposed to IL- $1 \beta$ for $24 \mathrm{~h}$ and intracellular vWF levels were determined by immunocytochemistry (Figure 5d). In the NEC cultures of all five patients the intracellular levels of vWF were significantly reduced after exposure to IL-1 $\beta$ (Figure 5d).

\section{DISCUSSION}

We established a protocol for the isolation of pure, viable EC cultures from CRC and healthy colon tissue. To avoid contamination of TEC cultures by NEC the tissue samples were obtained from a macroscopically non-hypoxic and non-necrotic central part of the tumor. We were aware that the invasion front represents the angiogenically most active part of the tumor. However, macroscopically it is not possible to determine the exact border between tumor and healthy tissue. Therefore, the use of samples derived from the tumor front might have increased the risk of contaminating TEC with NEC.

Isolation of microvascular EC from gliomas by Percoll gradient density centrifugation and/or magnetically labeled antibodies has already been described. ${ }^{19}$ For the isolation of EC from the colorectal carcinoma an approach with magnetically labeled antibodies against CD31 and CD105 was chosen. However, CD105 is also expressed on tumor stromal components. ${ }^{20}$ In fact, using this antigen for the isolation of EC no pure cultures could be established. In contrast, using CD31-MicroBeads EC cultures with a high purity (>93\%) could be obtained as demonstrated by FACS analysis. FACS results for CD31 staining did not yield 100\% because background signals observed in the isotype stainings were subtracted. However, comparison of the results obtained with TEC and NEC ( $>93 \%$ purity) with those from commercially available HUVEC ( $94.68 \%$ purity) showed that almost $100 \%$ pure EC cultures were obtained.

All of the established cultures formed capillaries in a basement membrane extract, bound Ulex europaeus lectin, took up ac-LDL and were uniformly positive for CD31, CD105 and VE-cadherin. The isolated cells were able to express inducible endothelial adhesion molecules such as VCAM-1, ICAM- 1 and E-selectin upon IL- $1 \beta / \mathrm{TNF}-\alpha$ stimulation. Contamination of the cultures by other cells 

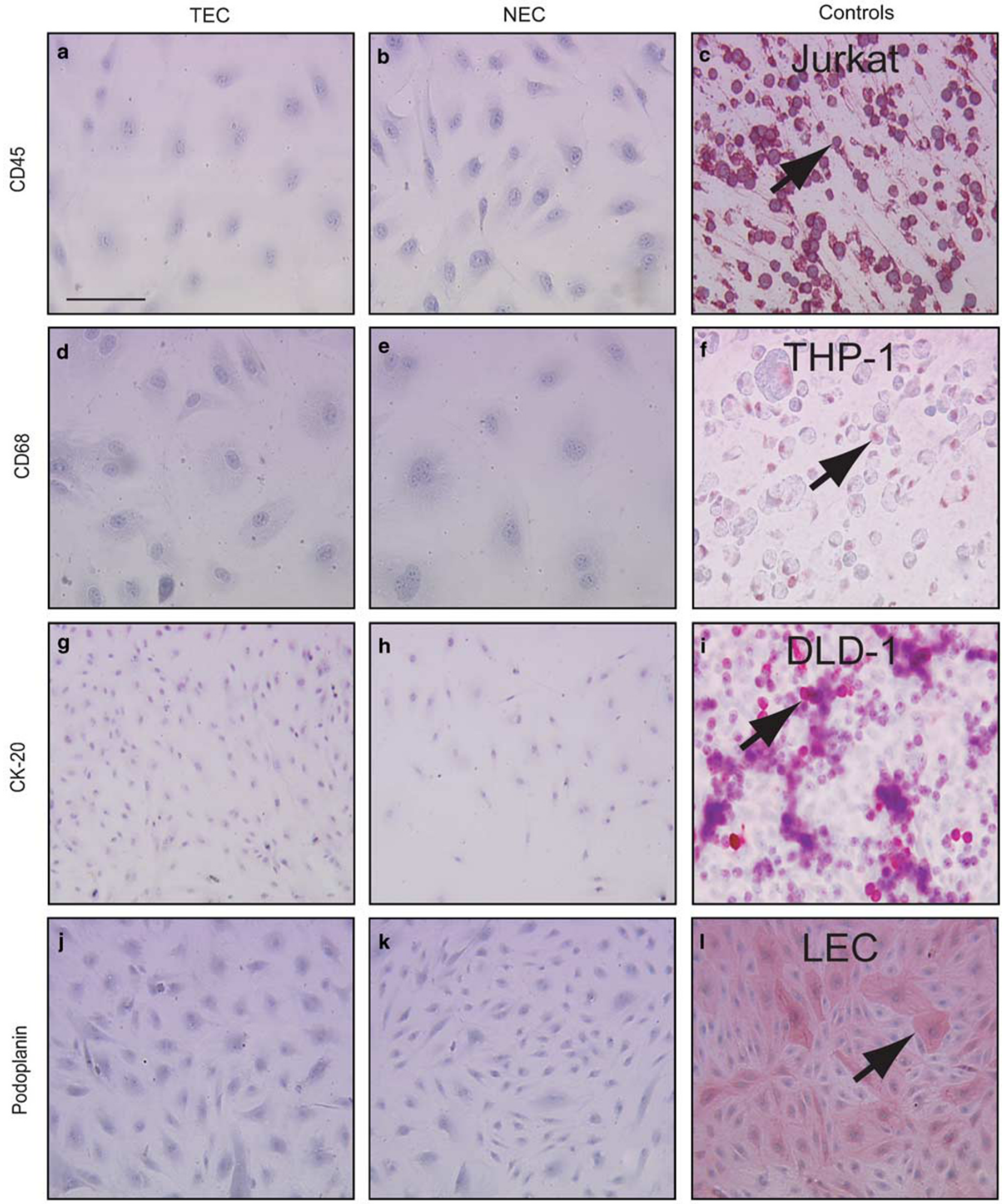

Figure 4 Tumor endothelial cells (TEC) and normal endothelial cells (NEC) are negative for CD45, CD68, cytokeratin-20 and podoplanin. TEC and NEC were immunocytochemically stained for (a and b) CD45 (hematopoietic cell marker), (d and e) CD68 (monocytes/macrophages), (g and h) cytokeratin-20 (CK-20, colonic epithelial cells) and (j and k) podoplanin (lymphatic endothelial cells). Positive controls: (c) CD45: Jurkat (lymphocyte cell line), (f) CD68: THP-1 (acute monocytic leukemia cell line), (i) CK-20: DLD-1 (colorectal carcinoma cell line) and (I) podoplanin: primary lymphatic endothelial cells = LEC. Positive staining is visualized by pink color (arrows). Counterstaining was performed with hematoxylin (blue color). Scale bar corresponds to $50 \mu \mathrm{m}$. 
a
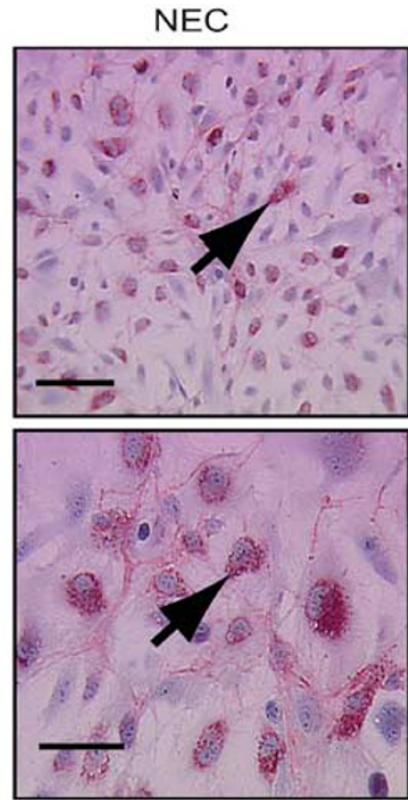

c

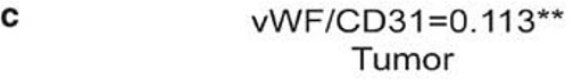

TEC
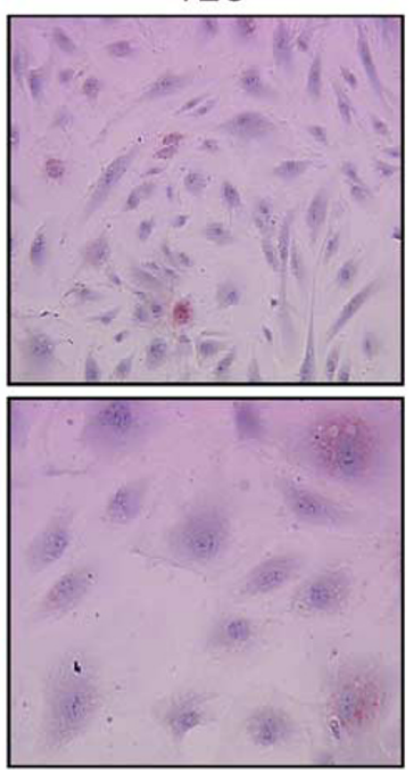

b

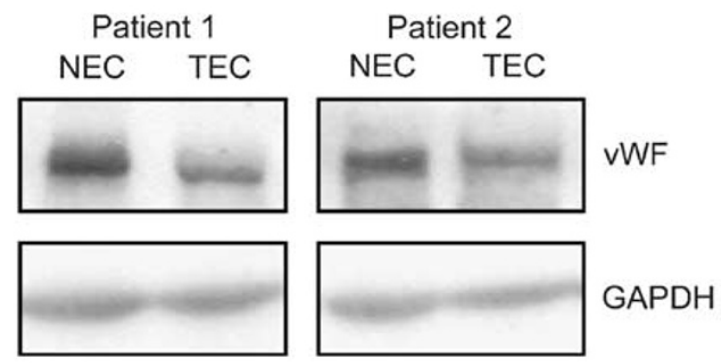

$v W F / C D 31=0.758$ Mucosa
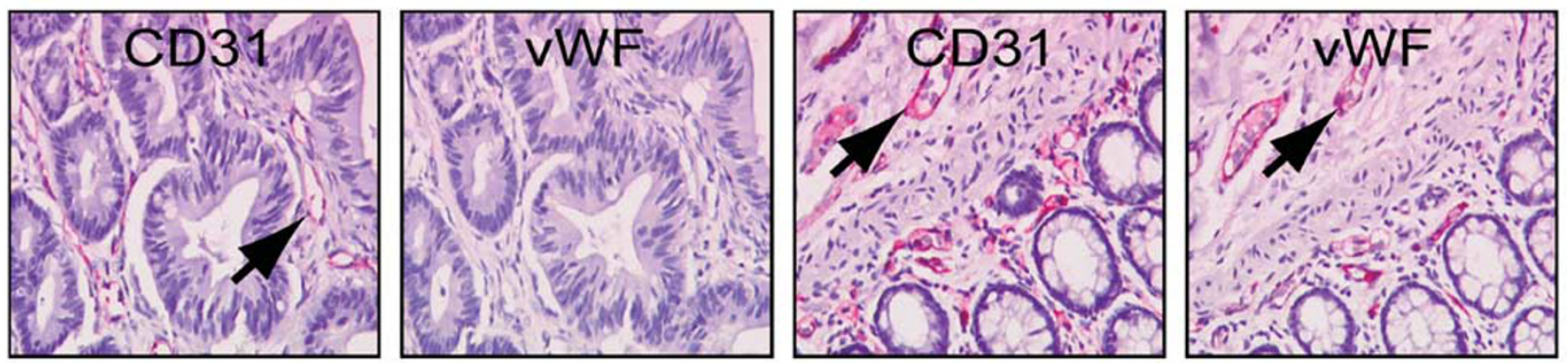

d

NEC 1
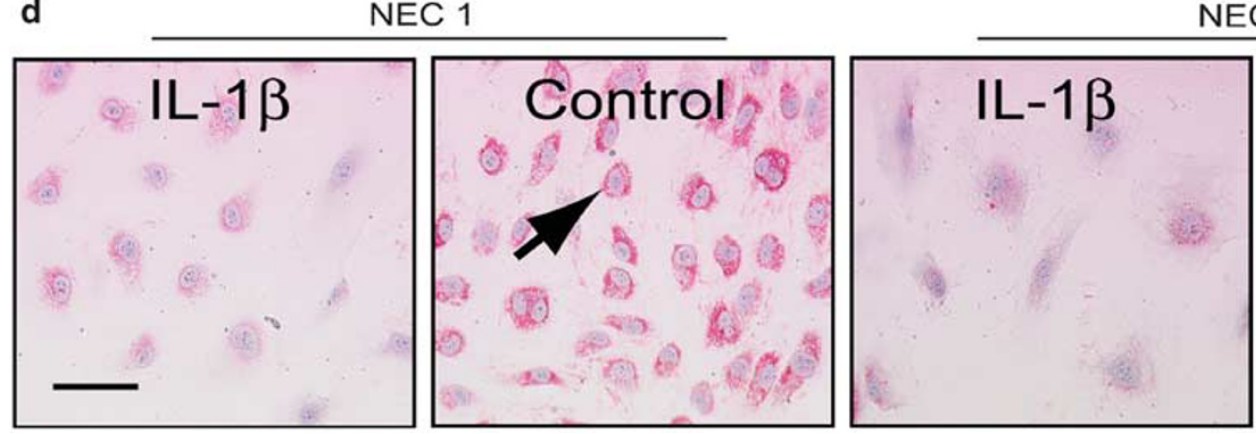

NEC 2

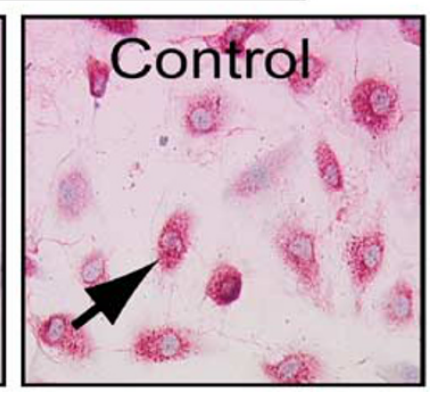

Figure 5 Tumor endothelial cells (TEC) from colorectal carcinoma show reduced intracellular von Willebrand factor (vWF) levels as compared to normal endothelial cells (NEC), both in vitro and in vivo. (a) Endothelial cell (EC) isolated from TEC and NEC were stained for vWF by immunocytochemistry. Positive staining is visualized by pink color (arrows). Counterstaining was performed with hematoxylin (blue color). Scale bar corresponds to $100 \mu \mathrm{m}$ (upper pictures). (b) VWF western blot analysis of total cell lysates from TEC and NEC cultures of two different patients (vWF molecular weight approximately $300 \mathrm{kDa}$ ). GADPH was stained to demonstrate equal amounts of protein loaded. (c) Immunohistochemical staining of CD31 and vWF was performed on consecutive sections of patients with differential levels of vWF in the corresponding TEC/NEC cultures. Tumor and normal tissue was stained. The ratios of the average number of VWF- and CD31-positive vessels were calculated, both from the center of the tumor and the normal tissue. Highly significant differences are indicated by two asterisks. (d) NEC cultures from patients with differential levels of intracellular vWF were either unstimulated (control) or treated with interleukin (IL)-1 $\beta$ for $24 \mathrm{~h}$ (IL-1 $\beta$ ) and stained for intracellular vWF expression by immunocytochemistry. Positive staining is visualized by pink color (arrows). Counterstaining was performed with hematoxylin (blue color). Scale bar corresponds to $50 \mu \mathrm{m}$. 
such as hematopoietic cells, monocytes/macrophages or colonic epithelial cells was excluded by staining of CD45, CD68 and cytokeratin-20. The cultures were not of lymphatic endothelial origin as shown by negative podoplanin staining. Altogether, this demonstrates convincingly the successful isolation of viable EC of blood vessel origin from the colorectal carcinoma and normal colon tissues.

Previously, differences in gene expression of non-cultivated NEC and TEC of CRC were described by St Croix et al. ${ }^{10}$ Until now, comparison of gene expression of cultivated viable, pure EC freshly isolated from tumor and healthy colon tissues have not been performed. Therefore, it will be interesting to determine whether the specific tumor endothelial markers (TEMs) shown to be associated with TEC shortly after isolation by St Croix are persistently expressed in long-term cultures of TEC.

To detect differences that might be induced by the different microenvironments to which TEC and NEC are exposed in vivo and may be stably maintained in the cultivated EC we analyzed the presence of vWF. This protein is stored within the Weibel-Palade bodies of EC and is released by the presence of inflammatory cytokines such as IL-1 $\beta$ and TNF- $\alpha .{ }^{21}$ Inflammation is commonly associated with CRC. In addition, increased serum levels of vWF have been reported in CRC patients and have been related to the progression of tumor and dissemination of malignant cells. ${ }^{22,23}$ Remarkably, vWF was absent or only weakly expressed in all of the eight TEC cultures, but was highly expressed in five of the corresponding NEC cultures. NEC of three patients displayed only weak levels of vWF.

These results were confirmed by western blot analysis of total cell lysates. Moreover, also in vivo there was a striking difference in the absolute numbers of vWF-positive vessels in the central part of the tumor and the normal mucosa. The ratio of vWF- $v s$ CD31-positive vessels was 6.7-fold lower in the tumor (tumor ratio $=0.113$; normal mucosa ratio $=0.758)$ demonstrating a strong decrease of intracellular vWF concentrations in blood vessels of colorectal carcinoma. These results suggested that the downregulation of intracellular vWF concentration in TEC in vivo is stably perpetuated by the isolated TEC in the in vitro culture.

It has been suggested that senescence may reduce vWF concentrations in ECs isolated from the kidney. ${ }^{24}$ However, the reduced number of vWF-positive vessels in the CRC suggested that this effect was not related to senescence induced by in vitro passaging but to the downregulation of intracellular vWF concentrations by the microenvironment in the tumor.

To investigate which mechanisms may be responsible for the reduced intracellular vWF levels different NEC cultures were treated with IL-1 $\beta$. In this experiment, we observed a similar reduction of intracellular vWF levels in the isolated NEC as compared to TEC of the same patients. This indicated that lower intracellular concentrations of vWF in TEC as compared to NEC may be induced by the inflammatory stroma reaction in CRC in vivo and are stably perpetuated by the cultivated cells in vitro.

Altogether, we provide the first protocol for the successful isolation of viable EC from CRC and healthy colon tissues. These cultures may be a valuable tool to identify functional differences of TEC and NEC and to optimize antiangiogenic treatment strategies of CRC.

\section{ACKNOWLEDGEMENT}

We thank Gertrud Hoffmann, Christina von Kleinsorgen and Melanie Nurtsch for excellent technical support and all patients participating at this study. We also thank Professor Papadopoulos and Dr Dimmler (Department of Pathology) for kindly providing the histology reports. This work was supported by grants from the ELAN-program of the University Hospital of the Friedrich-Alexander-University of Erlangen-Nuremberg to EN and RSC (no. 05.06.05.1), grants from the Doktor Robert Pfleger Stiftung Bamberg to VS and EN and from the interdisciplinary center of clinical research (IZKF) of the University of Erlangen to MS.

1. Quesada AR, Munoz-Chapuli R, Medina MA. Anti-angiogenic drugs: from bench to clinical trials. Med Res Rev 2006;26:483-530.

2. Jain RK. Normalization of tumor vasculature: an emerging concept in antiangiogenic therapy. Science 2005;307:58-62.

3. Folkman J. Tumor angiogenesis: therapeutic implications. N Engl J Med 1971;285:1182-1186.

4. Folkman J. Angiogenesis. Annu Rev Med 2006;57:1-18.

5. Carmeliet P. VEGF as a key mediator of angiogenesis in cancer. Oncology 2005;69(Suppl 3):4-10.

6. Zanetta L, Marcus SG, Vasile J, et al. Expression of von Willebrand factor, an endothelial cell marker, is up-regulated by angiogenesis factors: a potential method for objective assessment of tumor angiogenesis. Int J Cancer 2000;85:281-288.

7. Klagsbrun M. Mediators of angiogenesis: the biological significance of basic fibroblast growth factor (bFGF)-heparin and heparan sulfate interactions. Semin Cancer Biol 1992;3:81-87.

8. Jain RK. Normalizing tumor vasculature with anti-angiogenic therapy: a new paradigm for combination therapy. Nat Med 2001;7:987-989.

9. Jain RK. Molecular regulation of vessel maturation. Nat Med 2003;9:685-693.

10. St Croix B, Rago C, Velculescu V, et al. Genes expressed in human tumor endothelium. Science 2000;289:1197-1202.

11. Hida K, Klagsbrun M. A new perspective on tumor endothelial cells: unexpected chromosome and centrosome abnormalities. Cancer Res 2005;65:2507-2510.

12. Saunders $M$, Iveson T. Management of advanced colorectal cancer: state of the art. Br J Cancer 2006;95:131-138.

13. Hurwitz $\mathrm{H}$, Kabbinavar $\mathrm{F}$. Bevacizumab combined with standard fluoropyrimidine-based chemotherapy regimens to treat colorectal cancer. Oncology 2005;69(Suppl 3):17-24.

14. Hurwitz H, Fehrenbacher L, Novotny $W$, et al. Bevacizumab plus irinotecan, fluorouracil, and leucovorin for metastatic colorectal cancer. N Engl J Med 2004;350:2335-2342.

15. Lubeseder-Martellato C, Guenzi E, Jorg A, et al. Guanylate-binding protein-1 expression is selectively induced by inflammatory cytokines and is an activation marker of endothelial cells during inflammatory diseases. Am J Pathol 2002;161:1749-1759.

16. Naschberger E, Lubeseder-Martellato C, Meyer N, et al. Human guanylate binding protein-1 is a secreted GTPase present in increased concentrations in the cerebrospinal fluid of patients with bacterial meningitis. Am J Pathol 2006;169:1088-1099.

17. Guenzi E, Topolt K, Cornali E, et al. The helical domain of GBP-1 mediates the inhibition of endothelial cell proliferation by inflammatory cytokines. EMBO J 2001;20:5568-5577.

18. Guenzi E, Topolt K, Lubeseder-Martellato C, et al. The guanylate binding protein-1 GTPase controls the invasive and angiogenic capability of endothelial cells through inhibition of MMP-1 expression. EMBO J 2003;22:3772-3782. 
19. Miebach S, Grau S, Hummel V, et al. Isolation and culture of microvascular endothelial cells from gliomas of different WHO grades. J Neurooncol 2006;76:39-48.

20. Fonsatti E, Altomonte M, Nicotra MR, et al. Endoglin (CD105): a powerful therapeutic target on tumor-associated angiogenetic blood vessels. Oncogene 2003;22:6557-6563.

21. Giddings JC, Shall L. Enhanced release of von Willebrand factor by human endothelial cells in culture in the presence of phorbol myristate acetate and interleukin 1. Thromb Res 1987;47:259-267.
22. Damin DC, Rosito MA, Gus $\mathrm{P}$, et al. Von Willebrand factor in colorectal cancer. Int J Colorectal Dis 2002;17:42-45.

23. Wang Q, Shorten D, Xu X, et al. Effect of von Willebrand factor on the pharmacokinetics of recombinant human platelet glycoprotein Ibalpha-immunoglobulin G1 chimeric proteins. Pharm Res 2006;23:1743-1749.

24. Johnson TE, Umbenhauer DR, Hill R, et al. Karyotypic and phenotypic changes during in vitro aging of human endothelial cells. J Cell Physiol 1992;150:17-27. 